\title{
Diagnóstico de la industria maderera en Veracruz
}

\author{
Laura C. Ruelas Monjardín 1 \\ Juan M. Chávez Cortes ${ }^{2}$
}

\begin{abstract}
RESUMEN
Se analiza la situación de la industria maderera de Veracruz, haciendo un análisis de la que está instalada en la región centrooriente del país, integrada por los estados de Puebla, México, Tlaxcala e Hidalgo. Para ubicar la situación de la industria de Veracruz en el contexto nacional, se compara ésta con la de los tres principales estados productores de madera, como son Durango, Chihuahua y Michoacán. La comparación con la región centro-oriente obedece a que por razones geográficas, constituye el mercado natural para los productos de Veracruz. Para el análisis de dicha región se tomó como base la información de las encuestas que aplicó la SEMARNAP durante el período septiembrediciembre de 1995 a la industria forestal del país. Se evaluaron también el número de industrias, municipios en que están asentados, sector al que pertenecen, capacidad industrial instalada $y$ aprovechada y giro industrial. Se evaluó el potencial que tiene la industria, para aprovechar el incremento anual del volumen de los bosques y selvas. Para Veracruz, se hizo una comparación de los precios de los productos maderables y la relación que guardan con el promedio nacional, tomando en cuenta los puntos de venta, como son: en pie, en brecha y en planta. Los
\end{abstract}

resultados muestran que la industria, formada principalmente por aserraderos, opera en un $39 \%$ de su capacidad y que Veracruz ha venido experimentando una baja en su producción. Un factor que ha influido en esto, es sin duda, la conversión de su territorio al uso agropecuario, principalmente a la ganadería.

\section{PALABRAS CLAVE:}

Industria forestal, capacidad industrial instalada, capacidad industrial aprovechada, giro industrial, precios de la madera.

\section{ABSTRACT}

An analysis about the status of the wood industry in the state of Veracruz, México is done, simultaneously, it is assessed that established in the states of Puebla, México, Tlaxcala and Hidalgo. For the objectives of this paper, these states are considered to be grouped in the mid-west region. Also, to outline the situation of Veracruz in the national context, it is compared with the most important states regarding wood production, which are: Durango, Chihuahua and Michoacán. The relationship with the midwest region is that, because of geographical reasons, the region becomes the natural market for Veracruz forest products. The analysis was based in a survey applied by the Secretary of the Environment, Natural

1 Departamento de Productos Forestales y Conservación de Bosques, Instituto de Ecología, A.C., Apdo. Postal 63, 91000 Xalapa, Ver. México.

2 Departamento del Hombre y su Ambiente. Universidad Autónoma Metropolitana. Unidad Xochimilco. Calzada del Hueso 1100, Col. Villa Quietud. 04960 México, D.F. Manuscrito recibido para su publicación el 4 de Diciembre de 1996 
Resources and Fisheries, to the national forest industry, during September-December 1995. This diagnostic includes: number of industries, municipalities in which they are located, type of ownership, industrial capacity and its current state of use and type of industry. The industry potential is assessed, taking into account the forest land and the volume that could be exploited per state and for the region. For the state of Veracruz the level of wood prices are given, comparing them with the national average, and taking into account the sale point: standing, in the road and in the yard. Survey's results point out that, the sawing industry prevails; the average industry works at $39 \%$ of its capacity. Since the 50 's, wood industry production in Veracruz has been falling and its industrial capacity is underutilized. The situation could be partially explained by the conversion of forestlands to agricultural uses, mainly for cattle ranching.

\section{KEY WORDS:}

Forest industry, industrial capacity installed, industrial capacity exploited, type of industry, wood products prices.

\section{INTRODUCCION}

La contradicción central que esta investigación aborda, es la de que el bosque, hasta la fecha, no representa un recurso rentable para sus propietarios, ya que no ha sido una opción de ingresos económicos ni de mejora en la calidad de vida para los mismos.

Los datos actuales y aquellos que datan de 1951, demuestran que la producción de madera ha ido a la baja y que ésta se ha concentrado en el Norte del país. En el período 1952-1954, Chihuahua y Durango aportaban el $61.6 \%$ de la producción nacional, seguidos por los estados de Guerrero, Oaxaca y Michoacán, con el $21.8 \%$. Por otro lado, la situación para Veracruz era desfavorable, debido a la veda impuesta; ya que para ese mismo período, la producción disminuye $81 \%$ (Gallant, 1958). Dos décadas después, de acuerdo con De la Cueva (1978), el 64\% de la producción nacional fue aportado por los estados de Chihuahua, Durango y Michoacán. Veracruz sólo aportó el 1.9\%. Para 1994, estos tres estados aportaron el $62.6 \%$ y Veracruz el $1.05 \%$ (SAGAR, 1996).

Entre los factores que pueden explicar esta persistente baja en el aprovechamiento forestal, está el desplazamiento que han experimentado las áreas forestales por la actividad agropecuaria, de manera particular, por la ganadería. En 1940, la superficie de pastos ocupaba 1.6 millones de ha $(21.6 \%)$ y la de bosques 2.2 millones de ha $(30.9 \%)$. La población bovina en Veracruz, representaba el $7.4 \%$ de las existencias nacionales, lo que ubicaban al estado en el tercer lugar nacional. Para 1950 la superficie de pastizales representó el $25.5 \%$ de la superficie total del estado, la población bovina ocupó el segundo lugar nacional, con el $8.7 \%$. Se observa que el ritmo de crecimiento de la ganadería bovina en el periodo 1940-1950 registró una tasa media anual de $71 \%$. Para 1978, el estado ocupaba el primer lugar nacional, con $4,582,368$ cabezas, con el $14.4 \%$ del total nacional (Fernández et al., 1993).

La disminución de la importancia de las actividades forestales es un hecho que ocurre en todo el país. De acuerdo con Chapela y Mendoza (1996) la industria forestal nacional, contribuye con poco menos del $1 \%$ al Producto Interno Bruto (PIB) y tiende a disminuir por una baja interna en la producción; así como por un crecimiento mayor en el sector industrial y de servicios. En un período de 13 años (1980-1993) su contribución al PIB nacional cayó en un 31\%. Esto es, en 1980 participó con el $1.36 \%$ y en 1993 lo hizo con sólo el $0.94 \%$. Aún más dramáticas son las cifras por sector, ya que dentro del PIB agropecuario, la industria forestal descendió 
$65 \%$ en 23 años (1960-1993). Con respecto al PIB manufacturero, cayó $77 \%$ en el mismo lapso, puesto que en 1960 contribuyó con el $12 \%$ y en 1993 lo hizo con el 2.8\% (V Informe Presidencial, citado en Chapela y Mendoza, 1996).

Por otro lado, las industrias forestales en el país contribuyen poco a resolver las demandas nacionales de productos forestales. México es actualmente uno de los principales importadores de insumos forestales, dado que en la última década, y dentro del contexto de Latinoamérica, fue el segundo mayor importador de madera aserrada $\left(0.6\right.$ millones de $\left.\mathrm{m}^{3}\right)$, tableros de madera $\left(0.26\right.$ millones de $\left.\mathrm{m}^{3}\right)$ y pulpa de madera ( 0.3 millones de toneladas. También fue significativa la importación de papel de desperdicio para reciclaje en la industria del papel, con 1 millón de toneladas (Wardle, 1993). Es común encontrar a lo largo del país, instalaciones muy por abajo de la productividad forestal potencial y de la demanda actual de productos forestales en las diferentes regiones de México.

La región centro-oriente del país, objeto de esta investigación, se conformó para estos fines, por los estados de Hidalgo, México, Puebla, Tlaxcala y Veracruz. Esta región, con 3,703,817 ha de superficie arbolada, en conjunto, representa el $6.5 \%$ de la superficie forestal del país y el $6.45 \%$ de las existencias volumétricas, esto es $180,997,976 \mathrm{~m}^{3}$ rollo. Desde el punto de vista de la biodiversidad, esta región es también importante; entre otras cosas, porque alberga la tercera parte del bosque mesófilo que aún existe en el país.

Se tomó al estado de Veracruz como el eje de la presente investigación, ya que de este grupo de estados, es el más importante en recursos forestales. A nivel nacional ocupa el $3.2 \%$ de la superficie arbolada. La razón de incluir los otros estados radica en el hecho de que constituyen los mercados naturales, por razones geográficas, para los productos forestales de Veracruz.

En la región centro-oriente, la industria tiene una capacidad instalada para aprovechar $1,797,979 \mathrm{~m}^{3} / \mathrm{rollo}$, esto es para aprovechar escasamente el $1 \%$ de las existencias volumétricas. Por otro lado, si se toma el incremento anual del volumen forestal, que para la región asciende a $3,939,562 \mathrm{~m}^{3}$ rollo (Tabla 1), la industria tiene capacidad para aprovechar el $67.95 \%$ (Tabla 2).

Por otro lado, más de la mitad de la industria, el $57.35 \%$, se concentra en aserraderos, lo cual indica el escaso nivel de procesamiento que se lleva a cabo. En este trabajo se pretende demostrar, mediante un análisis comparativo, que los recursos forestales no son aún considerados como una opción para el desarrollo económico regional. Para este caso, el énfasis en la comparación se pondrá en los datos sobre recursos potenciales forestales y la capacidad industrial forestal de la región centro-oriente del país. A fin de evaluar el nivel de ingresos derivados de las actividades forestales, además, se comparará el nivel que guardan los precios del estado de Veracruz, con respecto al promedio nacional, tomando en cuenta los puntos de venta, como son: en pie, en brecha y en planta (Tablas 3 y 4).

\section{METODOS}

El origen de la información empleada son fuentes secundarias proporcionadas por SEMARNAP, resultado de una encuesta que aplicó a la industria forestal del país. La información proporcionada abarca el período septiembre-diciembre de 1995. 
Tabla 1. Superficie de bosques y selvas e incremento anual en volumen de la región CentroOriente del país

\begin{tabular}{|c|c|c|c|c|c|}
\hline ENTIDAD & $\begin{array}{c}\text { SUPERFICIE } \\
\text { DE } \\
\text { BOSQUES } \\
\text { (ha) }\end{array}$ & $\begin{array}{c}\text { SUPERFICIE } \\
\text { DE SELVAS } \\
\text { (ha) }\end{array}$ & $\begin{array}{l}\text { INCREMENTO } \\
\text { ANUAL EN } \\
\text { VOLUMEN DE } \\
\text { BOSQUES } \\
\text { ( } \mathrm{m}^{3} \text { rollo) }\end{array}$ & $\begin{array}{l}\text { INCREMENTO } \\
\text { ANUAL EN } \\
\text { VOLUMEN DE } \\
\text { SELVAS } \\
\text { (m³ rollo) }\end{array}$ & $\begin{array}{c}\text { INCREMENTO } \\
\text { TOTAL } \\
\text { (m } \mathrm{m}^{3} \text { rollo) }\end{array}$ \\
\hline Veracruz & 478,618 & $1,355,721$ & 301,643 & $1,355,721$ & $1,657,364$ \\
\hline Puebla & 460,771 & 307,455 & 564,901 & 307,455 & 872,356 \\
\hline Hidalgo & 230,743 & 172,942 & 212,374 & 172,942 & 385,316 \\
\hline México & 558,069 & 87,789 & 875,655 & 87,789 & 963,444 \\
\hline Tlaxcala & 51,709 & 0 & 61,082 & 0 & 61,082 \\
\hline TOTAL & $1,779,910$ & $1,923,907$ & $2,015,655$ & $1,923,907$ & $3,939,562$ \\
\hline
\end{tabular}

Fuente: SFFS, 1994

Tabla 2. Capacidad de la industria de la región Centro-Oriente para aprovechar los incrementos anuales en volumen

\begin{tabular}{|c|c|c|c|c|}
\hline \multirow[t]{2}{*}{ ENTIDAD } & \multirow{2}{*}{$\begin{array}{l}\text { CAPACIDAD } \\
\text { INDUSTRIAL } \\
\text { INSTALADA } \\
\text { (m³ rollo })\end{array}$} & \multirow{2}{*}{$\begin{array}{l}\text { CAPACIDAD } \\
\text { INDUSTRIAL EN } \\
\text { OPERACIÓN } \\
\text { (m³ rollo })\end{array}$} & \multicolumn{2}{|c|}{$\begin{array}{l}\text { APROVECHAMIENTO INDUSTRIAL } \\
\text { DEL INCREMENTO ANUAL DE } \\
\text { BOSQUES Y SELVAS (porcentaje) }\end{array}$} \\
\hline & & & Potencial & Real \\
\hline Veracruz & 172,100 & 41,140 & 10.38 & 2.48 \\
\hline Puebla & 366,021 & 137,075 & 41.95 & 15.71 \\
\hline Hidalgo & 188,330 & 60,974 & 48.87 & 15.82 \\
\hline México & 988,508 & 644,015 & 102.60 & 66.84 \\
\hline Tlaxcala & 83,020 & 58,340 & 135.91 & 95.51 \\
\hline Total & $1,797,949$ & 941,544 & 67.95 & 39.27 \\
\hline
\end{tabular}

Fuente: SEMARNAP, 1996b 
Tabla 3. Precios de la madera de Veracruz y precios promedio a nivel nacional

\begin{tabular}{|c|c|c|c|c|c|c|c|}
\hline \multirow[t]{2}{*}{$\begin{array}{l}\text { GRUPO DE } \\
\text { ESPECIES }\end{array}$} & \multirow[t]{2}{*}{ PRODUCTO } & \multicolumn{3}{|c|}{$\begin{array}{c}\text { PRECIO PROMEDIO } \\
\text { EN VERACRUZ } \\
\text { (pesos } / \mathrm{m}^{3} \text { rollo) }\end{array}$} & \multicolumn{3}{|c|}{$\begin{array}{c}\text { PRECIO PROMEDIO } \\
\text { NACIONAL } \\
\text { (pesos } / \mathrm{m}^{3} \text { rollo) }\end{array}$} \\
\hline & & En pie & Brecha & Planta & En pie & Brecha & Planta \\
\hline \multirow{3}{*}{ Pino } & ELD & & 176 & 222 & & 183 & 268 \\
\hline & ECD & & 111 & & & 104 & \\
\hline & PPM & & 103 & 162 & & 165 & 256 \\
\hline \multirow{3}{*}{$\begin{array}{l}\text { Otras } \\
\text { coníferas }\end{array}$} & ELD & & 176 & 222 & & 148 & 221 \\
\hline & ECD & & 111 & 162 & & 78 & 121 \\
\hline & PPM & & 103 & 162 & & 136 & 162 \\
\hline \multirow{4}{*}{$\begin{array}{l}\text { Otras } \\
\text { latifoliadas }\end{array}$} & ELD & & 82 & & & 107 & \\
\hline & ECD & & & & & & \\
\hline & PPM & & 82 & & & 343 & \\
\hline & Leña & & 20 & & & 62 & \\
\hline \multirow{3}{*}{$\begin{array}{l}\text { Maderas } \\
\text { preciosas }\end{array}$} & ELD & 270 & 495 & 631 & 242 & 503 & 743 \\
\hline & ECD & 190 & 310 & 430 & 139 & 339 & 450 \\
\hline & Contrachapados & 225 & 375 & 475 & 198 & 471 & 747 \\
\hline \multirow{5}{*}{$\begin{array}{l}\text { Comunes } \\
\text { tropicales }\end{array}$} & ELD & 80 & 150 & 200 & 63 & 160 & 314 \\
\hline & ECD & 40 & 70 & 90 & 41 & 142 & 209 \\
\hline & Chapa y contrachapados & 80 & 150 & 200 & 70 & 180 & 273 \\
\hline & PPM & & 40 & & & 196 & \\
\hline & Leña & 20 & 30 & 50 & 41 & 77 & 91 \\
\hline \multirow{5}{*}{ Encino } & ELD & & 90 & 125 & & 123 & 172 \\
\hline & ECD & & 40 & & & 69 & \\
\hline & Celulosa & & 40 & & & 56 & \\
\hline & PPM & & 68 & 125 & & 127 & 204 \\
\hline & Leña & & 50 & & & 71 & \\
\hline
\end{tabular}

\section{NOTACIONES:}

ELD = Escuadría dimensiones largas

$E C D=$ Escuadría dimensiones cortas

PPM = Postes, pilotes y morillos

Contrachapados $=$ Tableros de madera contrachapada (triplay) 
Tabla 4. Diferencias en los precios de Veracruz con el promedio nacional

\begin{tabular}{|c|c|c|c|c|c|c|c|}
\hline \multirow{3}{*}{$\begin{array}{c}\text { GRUPO } \\
\text { DE } \\
\text { ESPECIES }\end{array}$} & \multirow{3}{*}{ PRODUCTO } & \multicolumn{6}{|c|}{ DIFERENCIAS (pesos) } \\
\hline & & \multicolumn{3}{|c|}{ Veracruz } & \multicolumn{3}{|c|}{ Promedio nacional } \\
\hline & & En pie & Brecha & Planta & En pie & Brecha & Planta \\
\hline \multirow{3}{*}{ Pino } & ELD & & & & & 7 & 46 \\
\hline & ECD & & 7 & & & & \\
\hline & PPM & & & & & 62 & 96 \\
\hline \multirow{3}{*}{\begin{tabular}{|l} 
Otras \\
coníferas
\end{tabular}} & ELD & & 28 & 1 & & & \\
\hline & ECD & & 33 & 41 & & & \\
\hline & PPM & & & & & 33 & \\
\hline \multirow{4}{*}{\begin{tabular}{|l} 
Otras \\
Iatifoliadas
\end{tabular}} & ELD & & & & & 25 & \\
\hline & ECD & & & & & & \\
\hline & PPM & & & & & 261 & \\
\hline & Leña & & & & & 42 & \\
\hline \multirow{3}{*}{$\begin{array}{l}\text { Maderas } \\
\text { preciosas }\end{array}$} & ELD & 28 & & & & 8 & 112 \\
\hline & ECD & 51 & & & & 29 & 20 \\
\hline & Contrachapados & 27 & & & & 96 & 272 \\
\hline \multirow{5}{*}{$\begin{array}{l}\text { Comunes } \\
\text { tropicales }\end{array}$} & ELD & 17 & & & & 10 & 114 \\
\hline & ECD & & & & 1 & 72 & 119 \\
\hline & Chapa y contrachapados & 10 & & & & 30 & 73 \\
\hline & PPM & & & & & 156 & \\
\hline & Leña & & & & 21 & 47 & 41 \\
\hline \multirow{5}{*}{ Encino } & ELD & & & & & 33 & 47 \\
\hline & ECD & & & & & 29 & \\
\hline & Celulosa & & & & & 16 & \\
\hline & PPM & & & & & 59 & 79 \\
\hline & Leña & & & & & 21 & \\
\hline
\end{tabular}

3 La cantidad expresada en cada celda está indicando que en ese punto de venta el precio es más alto, con respecto al que se compara

Fuente: SEMARNAP, 1996b 
Para este estudio se tomaron en cuenta los datos reportados para los estados de Veracruz, Puebla, Tlaxcala, México e Hidalgo. Los conceptos que se analizaron fueron: giro industrial, sector al que pertenece, capacidad instalada y capacidad en operación. Aunque se consideró al municipio como la unidad geográfica, para el caso de Veracruz se analizará también el establecimiento de la industria por región, debido a que el estado, para fines forestales, ha sido dividido en cuatro regiones, que son: Cofre de Perote, Huayacocotla, Orizaba y Los Tuxtlas; sin embargo para esta última región, el Padrón no proporciona información, así como tampoco la da el Plan Sectorial Estatal.

Para determinar el nivel de aprovechamiento que podría tener la industria, de acuerdo con su capacidad instalada y la que se utiliza realmente, se analizaron las superficies forestales por estado, considerando sus bosques y selvas, sus existencias volumétricas y el incremento anual en volumen. Los datos de superficies forestales y de incrementos para bosques se tomaron del Inventario Nacional Forestal Periódico 1994 (SFFS, 1994), debido a que éste inventario que se inicia en 1992, es el primero en proporcionar incrementos en volumen -sólo para bosques- y que a diferencia del Inventario Nacional Forestal de Gran Visión (INFGV) de 1991, cuyos datos se tomaron como base para la elaboración del Compendio estadístico de la producción forestal 1989-1993, las cartas forestales se presentan a escala 1:250,000. De ahí que, los datos del INFGV sean diferentes de los que se presentan en este Inventario Periódico. Los muestreos de campo para la elaboración de este Inventario, dieron los resultados siguientes: en bosque de coníferas cerradas se obtuvo un incremento promedio anual de $1.86 \mathrm{~m}^{3}$ rollo por ha; en coníferas y latifoliadas cerradas, el incremento fue de $1.39 \mathrm{~m}^{3}$ rollo por ha; para los bosques abiertos de coníferas, el incremento fue de $1.38 \mathrm{~m}^{3}$ rollo por ha y para la mezcla de coníferas y latifoliadas se reportaron $1.22 \mathrm{~m}^{3}$ rollo por ha. Cabe mencionar que no se dan datos de incrementos para selvas; ante ello, recurrió a lo reportado por Jordan (1983), que es de $1 \mathrm{~m}^{3}$ rollo por ha.

Para el estado de Veracruz, se evaluaron los precios de los productos forestales y su relación con el promedio nacional. Los productos que se compararon fueron: escuadría en dimensiones cortas y largas, postes, pilotes y morillos, chapa y madera contrachapada ("triplay"), celulosa y leña y especies de madera, como pino (Pinus spp.), encino (Quercus spp.), maderas preciosas: cedro rojo (Cedrela odorata L.) y primavera (Roseodendron donell-smithii); comunes tropicales: ceiba (Ceiba petandra), palo de rosa o solerillo (Cordia alliodora Ruiz \& Pav. Cham.), hule (Castilla elastica Cerv.), napo, amargoso (Vatairea lundellii Standl. Kilip), verdecillo (Tabebuia chrysantha Jacq. Nicholson), lechoso; otras coníferas: ciprés (Cupressus benthamii Endl.); y otras latifoliadas como ilite (Alnus jorullensis Kunth), madroño (Arbutus spp.), liquidámbar (Liquidambar macrophylla Oersted), fresno (Fraxinus uhdei Wenz). Los costos fueron estimados en sus puntos de venta, como son: en pie, en brecha y en planta; y se estimó el nivel que guardan con respecto al promedio nacional. Se recalca que sólo se da para Veracruz, debido a que la información de las encuestas para los otros estados fue muy pobre.

Finalmente, en el análisis de datos sólo se aborda el enfoque de estadística descriptiva, debido a que se busca demostrar, que en país, ni el sector industrial considera al bosque como un recurso para su desarrollo, lo cual queda demostrado al comparar las capacidades reales y potenciales de la infraestructura. Aunque existen otros métodos, como el del cálculo del valor presente, pero que para los fines de este trabajo no es aplicable, debido 
a que no se pretende hacer una evaluación de las industrias forestales a la luz de sus rendimientos económicos de mediano y largo plazo, ni se está tratando de determinar el valor del bosque, frente a otras formas alternativas de uso de las tierras.

\section{RESULTADOS}

En primera instancia se presentarán los resultados por entidades, para posteriormente hacer una comparación interregional y en los casos en que se contó con información para el total nacional, se hará en este contexto.

\section{Estado de Veracruz}

Los datos de la Subdelegación de Recursos Naturales de la SEMARNAP en Veracruz, arrojan que 57 municipios de la entidad registraron aprovechamiento forestal para 1995. Sin embargo, en el Padrón de la Industria Forestal, sólo 29 municipios reportaron 43 industrias forestales (Fig. 1). Cruzando ambas informaciones, de los 57 municipios, sólo 20 están incluídos en el padrón. Una explicación puede ser que las industrias no respondieron el cuestionario; o bien, venden su madera en pie.

Distribucion geografica de la industria. Considerando la información del padrón, el $50 \%$ de la industria está concentrada en 4 municipios: Huayacocotla con el $17 \%$, Perote $15 \%$, Villa Aldama con el $9.8 \%$ y las Vigas con el $7 \%$. El restante está dispersa en municipios como: Acultzingo, $\mathrm{Cd}$. Mendoza, Maltrata, Nogales, Orizaba, Altotonga y otros. Si se regionaliza, se encuentra que: el 44\% está localizada en el Cofre de Perote, el 33\% en Orizaba y el $23 \%$ en Huayacocotla (Fig. 2). De acuerdo con las cifras, Orizaba se ubica en el segundo lugar, cuando una década atrás, representaba en superficie el $61.3 \%$ de la explotación forestal estatal (CEPES, 1986); aún más, en el Plan Sectorial (Gob. Edo. Veracruz, 1995), para 1993 contaba con 22 aserraderos y para 1995 sólo contaba con 13, en tanto que en el Padrón de SEMARNAP se registraron 14 . El $90 \%$ de la industria pertenece al sector social y el 10\% al privado. En giro industrial, el $34 \%$ son aserraderos, el $44 \%$ son aserraderos/taller de secundarios, el $15 \%$ taller de secundarios, el $5 \%$ fábrica de muebles y el restante $2.43 \%$ lo constituyen las fábricas.

Capacidad industrial que se utiliza. Cabe mencionar que sólo se utiliza el $23.90 \%$ de la capacidad instalada. Si se toma en cuenta que el estado tiene una superficie arbolada de 1,834,339 ha y un incremento anual en volumen (bosques más selvas) de 1,657,364 $\mathrm{m}^{3}$ rollo, la industria sólo aprovecha el $2.48 \%$ de este potencial.

El nivel de los precios de Veracruz. Con los datos de precios que se proporcionaron, se calculó el valor agregado que obtienen los productos al pasar de un punto de venta a otro, como es de pie a brecha y de brecha a planta; así como la diferencia, expresada en porcentaje, del precio que tiene la madera en Veracruz, con respecto al promedio nacional. En la tabla 3 se registran los precios de los productos en cada punto de venta y en la tabla 4 se dan las diferencias; cabe mencionar que esta diferencia se ubicó en la celda donde es más alto el precio; por ejemplo, el precio de la escuadría de pino en cortas dimensiones, es \$7.27 más alto en Veracruz que en el resto del país.

Los análisis de los datos de las tablas 3 y 4 , indican que:

a) La madera de pino, en general, es más barata en Veracruz que en el resto del 


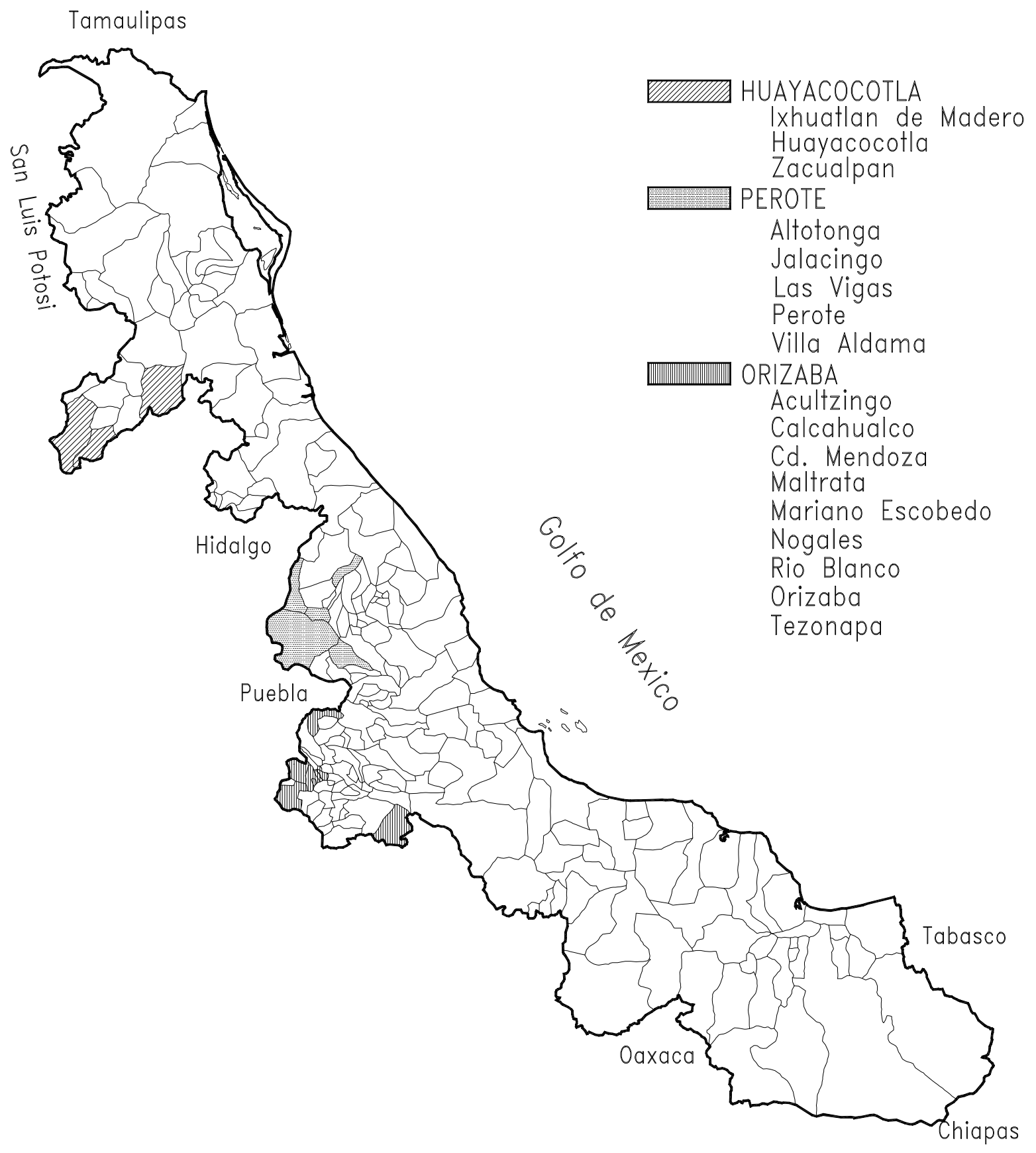

Figura 1. Distribución geográfica de la industria maderera en el estado de Veracruz 


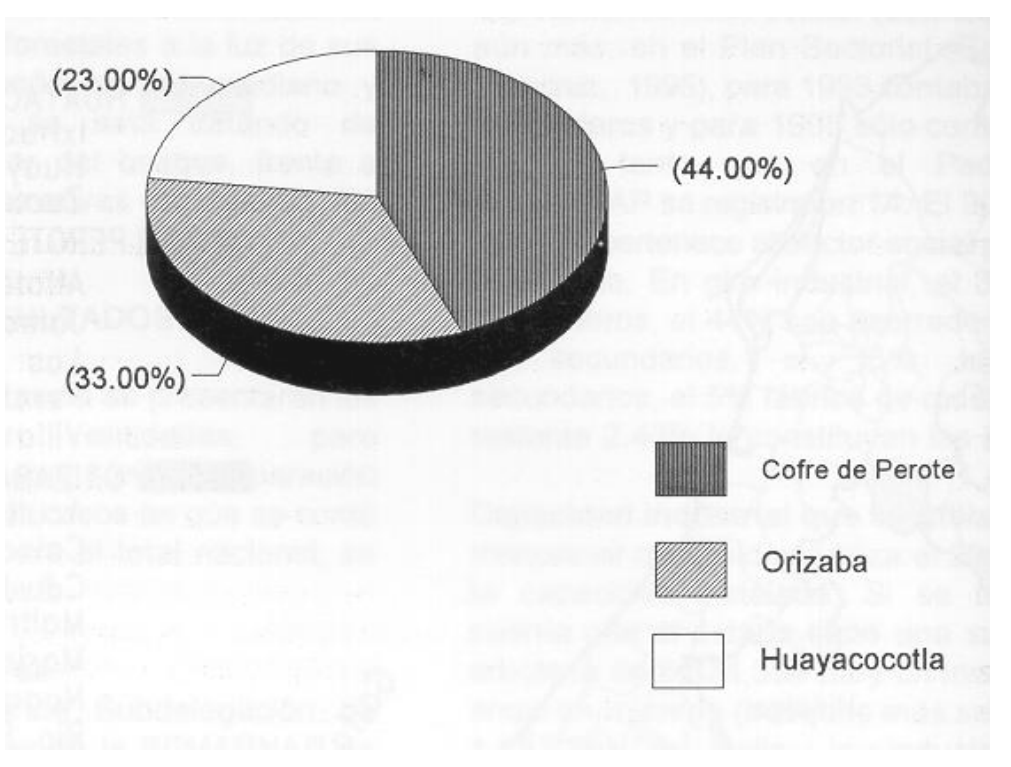

Figura 2. Distribución de la industria maderera en Veracruz

país; sobre todo los postes, pilotes y morillos puestos en planta, cuyo precio representa únicamente el $63 \%$ con respecto al promedio nacional.

b) La madera de otras coníferas, registró un precio más alto en Veracruz, el caso más sobresaliente fue el aumento del $30 \%$ de la escuadría de cortas dimensiones.

c) Los productos de otras latifoliadas, son más baratos en Veracruz; ya que los precios de los postes, pilotes y morillos, puestos en brecha son menores hasta en un $76 \%$ con respecto al promedio nacional.

d) Para el caso de las maderas preciosas -excepto las que se venden en pie- y las comunes tropicales, los precios en los otros puntos de venta son más bajos en Veracruz, por ejemplo, el precio de los postes, pilotes y morillos es menor hasta un $80 \%$, puesto en brecha, si se comparan con los precios promedio nacional.

Estos resultados, indican que el $100 \%$ de los productos analizados (un total de 22) se venden en brecha, de los cuales el $62.5 \%$ se vende también en planta y sólo el $29 \%$ lo hace en pie. De los grupos de especies, sólo las comunes tropicales y las preciosas se comercializan en pie. Grosso modo, el valor agregado que experimenta un producto, al variar su punto de venta en pie, a brecha, es de alrededor del $40 \%$, mientras que al pasar de brecha a planta aumenta un $23 \%$. Cabe mencionar, que en las tablas 3 y 4 se redondearon las cantidades a números enteros. 


\section{Estado de Hidalgo}

Esta entidad cuenta con 403,685 ha de superficie arbolada, existencias volumétricas de $17,264,283 \mathrm{~m}^{3}$ y tiene un incremento anual en volumen (bosques más selvas) de $385,316 \mathrm{~m}^{3}$. De acuerdo con el padrón, cuenta con 48 industrias forestales, de las cuales, el $54.16 \%$ está concentrada en los municipios de Acaxochitlán, Cuautepec, Pachuca, Metepec y Tulancingo. El 93.75\% está concentrada en el sector privado y el $6.25 \%$ en el social. Llama la atención el que 8 industrias estuvieran sin operar. De las 47 industrias que reportaron giro industrial, 41 son aserraderos y 6 son talleres de secundarios. La actividad de aserrío generó 366 empleos y la de taller de secundarios, 20. Sólo se utiliza el $32.37 \%$ de la capacidad instalada de la industria por lo que solo aprovecha el $15.82 \%$ de la productividad de su superficie forestal.

\section{Estado de Tlaxcala}

Este estado cuenta con 51,709 ha de superficie arbolada, existencias volumétricas de 4,030,441 $\mathrm{m}^{3}$ y una productividad total estimada de $61,082 \mathrm{~m}^{3}$ año. De acuerdo con el padrón, cuenta con 17 industrias forestales, las cuales están altamente concentradas en los municipios de Apizaco y Tlaxco, con el 76.47\%. De los 6 municipios que informaron sobre el establecimiento de un tipo de industria, el $82.35 \%$ está concentrado en el sector privado y el $11.76 \%$ en el sector social. El tipo de industrias asentadas son: aserraderos, aserraderos/fábrica, aserradero/fábrica de cajas, fábrica de cajas, fábrica de parquet, impregnadora y taller de secundarios. De los $83,020 \mathrm{~m}^{3}$ rollo de capacidad instalada, se encuentra en operación el $70.27 \%$ de la misma, esto es, $58,340 \mathrm{~m}^{3}$ al año. Esta entidad, junto con el Estado de México, cuenta con una industria cuya capacidad instalada, si operara a toda su capacidad, excedería el incremento anual de sus bosques. Aunque prácticamente aprovecha el $100 \%$ de este incremento, al registrar un $95.5 \%$ de aprovechamiento real.

\section{Estado de México}

Esta entidad, cuenta con 645,858 ha de superficie arbolada, $64,898,046 \mathrm{~m}^{3}$ en se encuentra preponderantemente en el sector privado, con el $93.50 \%$, el 5.19\% está en el sector social y el $1.29 \%$ en el sector educativo. El giro industrial más importante lo constituyen los aserraderos, con el $81.57 \%$ (62 unidades) del total, seguido por fábricas de triplay (5.26\%); taller de secundarios (5.26\%); fábrica de tableros (2.63\%); aserradero/fábrica (2.63\%); fábrica de cajas $(1.31 \%)$ y fábrica de celulosa $(1.31 \%)$. Su capacidad de operación real de $644,015 \mathrm{~m}^{3}$ rollo le permite utilizar el $66.84 \%$ del incremento anual del volumen. Aunque cabe mencionar que, su industria tiene la capacidad industrial instalada para aprovechar los incrementos anuales de su arbolado.

\section{Estado de Puebla}

La entidad, cuenta con 768,226 ha de superficie arbolada, 38,056,439 $\mathrm{m}^{3}$ de existencias volumétricas y un incremento anual en volumen de $872,356 \mathrm{~m}^{3}$. Tiene, tomando en cuenta el padrón, 89 industrias forestales, de las cuales el $50.53 \%$ está concentrada en los municipios de: Chignahuapan (21.34\%), San Martín Texmelucan (8.98\%), Zacapoaxtla (8.98\%) y Zacatlán (11.23\%). El $91.01 \%$ pertenece al sector privado y el $8.98 \%$ al sector social. En cuanto a giro industrial, el $40.69 \%$ son aserraderos, el 18.60 taller de secundarios, el $18.60 \%$ fábrica de cajas, el $17.44 \%$ aserradero/fábrica de cajas y el $4.65 \%$ aserradero/fábrica. En cuanto a la capacidad instalada, de $366,021 \mathrm{~m}^{3}$ rollo, sólo se utiliza el $37.45 \%\left(137,075 \mathrm{~m}^{3} / \mathrm{año}\right)$ lo que le permite sólo utilizar el $15.71 \%$ de su productividad estimada. Por último, cabe mencionar que los bosques de esta entidad, están entre los diez del país que tienen el 
mayor incremento en volumen, por lo que la industria aquí tiene ese potencial natural para su mejor desempeño.

\section{DISCUSION}

Los resultados antes expuestos, permiten estimar los bajos niveles de operación de las industrias, sobre todo para el estado de Veracruz. Si se considera que el potencial que tienen sus bosques y selvas, como se muestra en la tabla 1 , a pesar de que la región en su conjunto representa el $6.5 \%$ de la superficie forestal del país, su industria no ha sido capaz de explotar este potencial; la cual en conjunto, cuenta con una capacidad instalada de $1,797,979 \mathrm{~m}^{3}$ rollo, lo que significa que si la industria operara al $100 \%$ de su capacidad, sólo podría aprovechar, en promedio para la región, el $67.95 \%$ del incremento anual en volumen de los bosques y selvas. Retomando el caso de Veracruz, llama la atención que, a pesar de que cuenta con el $3.2 \%$ de la superficie arbolada del país, es la entidad con el menor aprovechamiento industrial, con sólo el $10.38 \%$ del potencial y la cifra se reduce aún más al $2.48 \%$, si se considera el aprovechamiento real. Aún cuando las cifras de aprovechamiento se duplicaran, el porcentaje de los volúmenes explotados, continuaría siendo insignificante. Ya que a juicio de los expertos, existe la tendencia entre los industriales de la madera, a proporcionar la mitad de lo que realmente utilizan (Rivera, 1996, Comunicación personal). Si a esto le añadimos el que, en cualquier encuesta, donde la gente tiene la opción de contestar o no, existe el potencial para el sesgo a la no respuesta (Forbes et al., 1993).

La situación crítica en el estado de Veracruz no es nueva, ya que, comparando un periodo crítico, 1979-1983, el aprovechamiento cayó en un $49.2 \%$, debido a que en 1979 el volumen de la producción forestal maderable fue de $110,320 \mathrm{~m}^{3}$ rollo, para 1983 fue de sólo $54,279 \mathrm{~m}^{3}$ rollo (SEMARNAP, 1996a).

Otro hecho, sin duda significativo, es el que la industria de la región, formada por 283 unidades, esté dominada por los aserraderos, los cuales representan el 55\% del giro industrial, con 155 unidades; esta proporción se incrementa aún más, si consideramos que otro $17 \%$ está formado por la unión de aserradero con taller de secundarios y fábricasy el otro $28 \%$ está atomizado en industrias de triplay, tableros, celulosa, impregnadora, fábrica de parquet, fábrica de muebles y talleres de secundarios (Tabla 5).

Esta situación no ha variado prácticamente en los últimos cinco años, ya que de acuerdo con datos de la CNIF (1991), de los $8,101,986 \mathrm{~m}^{3}$ rollo que se produjeron en 1990 en el país para fines industriales, el $67.72 \%$ se destinó a la industria del aserrío; industria que, vale la pena recalcar, en su gran mayoría es de carácter pequeño, ya que su capacidad instalada es menor de $25 \mathrm{~m}^{3} /$ aserrados por turno (Torres, 1990, citado en Flores et al., 1995).

En cuanto al número de industrias establecidas por estado, se aprecia que el estado de Puebla cuenta con el $43 \%$ del total regional, seguido por el estado de México con el $38 \%$; Hidalgo con el $23 \%$, seguido muy de cerca por Veracruz, con el $19 \%$ y finalmente Tlaxcala, con el $8 \%$. Aunque este último estado cuenta con menor número de industrias, el grado de diversificación de la misma es mayor, ya que es el único que cuenta con una fábrica de parquet, una impregnadora y prácticamente todos los tipos de industrias que se encontraron en los otros estados. Además, es la única entidad cuya industria aprovecha prácticamente el $100 \%$ de los incrementos anuales del bosque. Cabe mencionar que, el Estado de México 
Tabla 5. Caracterización de la industria forestal en la región centro-oriente del país

\begin{tabular}{|c|c|c|c|c|c|}
\hline GIRO INDUSTRIAL & VERACRUZ & PUEBLA & HIDALGO & MEXICO & TLAXCALA \\
\hline Aserradero/taller sec. & 20 & & & & \\
\hline Taller de secundarios & 6 & 16 & 6 & 4 & 3 \\
\hline Aserradero/ fca. cajas & & 15 & & & 4 \\
\hline Aserradero & 15 & 35 & 41 & 62 & 2 \\
\hline Aserradero/fábrica & & 4 & & 2 & 2 \\
\hline Fábrica de muebles & 1 & & & & \\
\hline Fábrica de cajas & 1 & 16 & 1 & & 4 \\
\hline Fábrica de parquet & & & & & 1 \\
\hline Impregnadora & & & & & 1 \\
\hline Celulosa & & 1 & & & \\
\hline Tableros & & 2 & & & \\
\hline Madera contrachapada & & 4 & & & \\
\hline TOTAL & 43 & 86 & 47 & 76 & 31 \\
\hline
\end{tabular}

Fuente: SEMARNAP, 1996a.

duplicó el número de plantas de aserraderos en un lapso de 5 años. En 1990 contaba con 34 unidades, excluyendo la zona de influencia de la fábrica de papel San Rafael (Flores et al., 1995) y para 1995 en el Padrón se registraron 62 unidades. Junto con el estado de Tlaxcala, es la entidad cuya industria también tiene capacidad para aprovechar los incrementos anuales. Además de que sus bosques de coníferas son de los que presentan, a nivel nacional, los mayores incrementos en volumen (SFFS, 1994).
Por lo tanto el panorama de la industria forestal en esta región y del país en general es pesimista, y cada vez más, es necesario el análisis de sus causas y del planteamiento de opciones para apoyar el desarrollo del sector forestal en el país.

\section{CONCLUSIONES}

El análisis a la industria de esta región, presenta un panorama pesimista, sobre todo para Veracruz, Puebla e Hidalgo, ya que si se considera que la superficie forestal es de $3,703,817$ ha y un incremento 
anual en volumen de los bosques y selvas de $3,939,562 \mathrm{~m}^{3}$ y la capacidad en operación de la industria es de $941,544 \mathrm{~m}^{3}$ rollo, sólo se está aprovechando el 39.27\% del incremento anual. Por lo que la conclusión general de que industria forestal aún está por debajo de las posibilidades de producción forestal se valida. Las explicaciones a esta situación puede ir desde datos falseados en la encuesta y en el poco o nulo suministro de información estadística por parte de los industriales; ya que existe poca disposición a proporcionar información, sobre todo cuando se solicita por correo. Sin embargo, ante las fuentes escasas de información al respecto, toda información que surja sobre la industria forestal debe ser difundida y analizada. Tal es el caso de la información procedente de SEMARNAP. Por lo que el diseño de nuevos instrumentos de generación y análisis de información sobre el sector, que den lugar a información verídica y real es urgente

\section{RECONOCIMIENTOS}

Se agradece a la Dirección General de Recursos Naturales de la SEMARNAP, el haber proporcionado la información de las encuestas para integrar el Padrón de la Industria Forestal Nacional; así como a al Ing. Luis Manuel Aguilar, de la Subdelegación de Recursos Naturales, SEMARNAP, en Veracruz por la información brindada.

Se agradece el apoyo de la C. Mónica Enríquez Magaña en la elaboración del mapa de la figura 2.

Este trabajo fue realizado con fondos fiscales asignados al Departamento de Productos Forestales y Conservación de Bosques, del Instituto de Ecología, A.C., con la clave 902-13.

\section{REFERENCIAS}

Cámara Nacional de la Industrial Forestal (CNIF). 1991. Memoria económica 1990-1991. México.

Chapela y Mendoza, G. 1996. Panorama del sector forestal en México. Gaceta Ecológica, Nueva época, No.38:27-39.

Centro de Estudios Políticos y Sociales (CEPES). 1986. Información básica. PRI, Comité Directivo Estatal Veracruz. Xalapa, Ver.

De la Cueva G., L. 1978. Análisis de la producción forestal en México. 19711976. Ciencia Forestal 3(12):22-39.

Fernández O., L.M., M. Tarrío G. y D. Villafuerte S. 1993. La expansión ganadera en Veracruz: sus impactos en la economía y en la producción de alimentos. In: N. Barrera e $\mathrm{H}$. Rodríguez, Coord. Desarrollo y medio ambiente en Veracruz. CIESAS-Golfo, Instituto de Ecología, A.C., Friedrich Ebert Stiftung, Xalapa, Ver. p:15-33.

Flores B., S., J.A. Torres P., J.L. Romo L. y F. Burguete H. 1995. El mercado de la madera aserrada en el Estado de México. Parte I. Abastecimiento e industria. Revista Chapingo, serie Ciencias Forestales 1(1)27-30.

Forbes, C.L., S.A. Sinclair y W.G. Luppold. 1993. Wood material use in the U.S. furniture industry: 1990 to 1992 . Forest Products Journal 43(7/8):59-65.

Gallant, M. 1958. El mercado de los productos forestales de México. In: Aprovechamiento de los recursos forestales. Informe presentado por la FAO al Banco de México, S.A. México, D.F. Tomo II. 
Gobierno del Estado de Veracruz y Secretaría del Medio Ambiente, Recursos Naturales y Pesca. 1995. Plan sectorial forestal del Estado de Veracruz (1996-2034). Documento extenso. Xalapa, Ver., México.

Jordan, C. 1983. Productivity in tropical rainforest ecosystems and the implications for their use as future wood and energy sources. In: Golley, F. (Ed.), Tropical rain forest ecosystem. Elsevier, Amsterdam. pp:117-136.

Rivera M., S. 1996. Comunicación personal. Noviembre de 1996.

Secretaría de Agricultura, Ganadería y Desarrollo Rural (SAGAR). 1996. Subsecretaría de Planeación. Anuario estadístico de la producción forestal 1994. México. 129p.

Secretaría del Medio Ambiente, Recursos Naturales y Pesca (SEMARNAP). 1996a. Subdelegación de Recursos
Naturales Veracruz. Informe del volumen y valor de la producción forestal maderable por grupo de especies según municipio. 1995. Xalapa, Ver.

Secretaría del Medio Ambiente, Recursos Naturales y Pesca (SEMARNAP). 1996b. Padrón de la industria forestal maderable en México, periodo septiembre-diciembre. México.

Subsecretaría Forestal y de la Fauna Silvestre (SFFS). 1994. Inventario Nacional Forestal Periódico, México 1994. Memoria Nacional. México. Secretaría de Agricultura y Recursos Hidraúlicos. 81p.

Wardle, P.A. 1993. Estadísticas sobre comercio de productos forestales en América Latina. In: FAO. Memoria, Seminario de estadísticas forestales para América Latina y el Caribe. Santiago, Chile, 19-30 abril 1993. pp:127-131. 